\title{
Religiöse Diversität als Herausforderung für die Katholische Kirche
}

\author{
Regina Polak
}

The Catholic Church is challenged by religious diversity in much the same ways as secular institutions. The Church is a point of reference for ordinary believers and political actors alike and therefore has responsibility for working towards justice and peace inside the Church as well as outside of it, in the world. The topic of living together in diversity is therefore an important issue for the Catholic Church. This article analyses how the Catholic Church has dealt with religious diversity. Four perspectives structure this analysis: 1 . In which socio-religious context does religious diversity become a practical theological challenge for the Church? 2. What is the ecclesiastical and socio-political relevance of processes of religious diversification for the Church? 3. Which theological hermeneutics can the Church draw for a proper understanding and handling of religious diversity? 4. What could a successful approach to religious diversity inside the Church look like? The article draws on literature and on one empirical study of practical approaches to religious diversity in parishes in the Dioceses of Vienna.

Regina Polak, Associate Professor for Practical Theology and research on religion at the Department for Practical Theology at the Catholic Faculty at the University of Vienna. Main research fields: Religion and Migration, Transformation of the religious field in Europe, Spirituality, Values, fundamental theological questions of the transforming Church in Europe. Recent publications: Religion im Wandel. Transformation religiöser Gemeinschaften in Europa durch Migration. Interdisziplinäre Perspektiven, co-edited with Wolfram Reiss (Vienna 2015); Mission in Europa? Auftrag - Herausforderung Risiko (Innsbruck 2012).

\section{Einleitung}

Religiöse Diversität stellt auch die Katholische Kirche global und lokal vor neue Herausforderungen. Als politische Akteurin trägt sie Verantwortung für Gerechtigkeit und Frieden in der Welt, und die Frage nach dem Zusammenleben in (nicht nur) religiöser Vielfalt muss daher an oberster Stelle der Agenda der Katholischen Kirche stehen. Die Auseinandersetzung mit diesem Thema hat aber auch innere, d.h. theologische Gründe. Die Katholische Kirche versteht sich selbst als ,Zeichen und Werkzeug für die innigste Vereinigung mit Gott wie für die Einheit der ganzen Menschheit“" . Dies verpflichtet sie, als „Werkzeug“ einen

1 Lumen Gentium 1. 
Beitrag zum friedlichen und gerechten Zusammenleben in einer (religiös) diversen Gesellschaft zu erbringen. Als „Zeichen“ wiederum erhebt sie den Anspruch, selbst modellhaft diese „Einheit in Vielfalt“- dies ist theologisch mit dem Begriff „katholisch“ gemeint -zu leben. Wie schwierig dies im Inneren der Kirche ist, zeigen allerdings die innerkirchlichen Spannungen zwischen konservativen Bewegungen wie z.B. Opus Dei oder dem Neokatechumenat auf der einen und Reformbewegungen wie der Plattform Wir sind Kirche auf der anderen Seite. Die Kirche steht wie die Gesellschaft vor einem neuartigen Lernprozess. Mein Beitrag wird sich daher auf die Frage konzentrieren, wie die Katholische Kirche in ihrem Inneren mit religiöser Diversität umgeht. Dies geschieht aus einer praktisch-theologischen Perspektive: d.h. dass ich auf der Basis sozialwissenschaftlicher Befunde nach theologischen Hermeneutiken frage, die vorfindbare Praxis zu reflektieren. Dies geschieht aus vier Perspektiven:

(a) Welches ist der sozioreligiöse Kontext, in dem religiöse Diversität für die Katholische Kirche zur Herausforderung wird?

(b) Worin besteht die kirchen- und gesellschaftspolitische Relevanz religiöser Diversifizierungsprozesse für die Kirche?

(c) Welche theologischen Hermeneutiken stehen zum Verständnis sowie zum Umgang mit dieser Situation zur Verfügung?

(d) Wo gibt es Orte, an denen die Kirche gegenwärtig lernt, mit religiöser Diversität umzugehen?

Die Größe und Komplexität der Kirche lässt für alle diese Fragen nur erste und überaus begrenzte, noch dazu sozialwissenschaftlich kaum validierte Hypothesen formulieren. Der Fokus der empirischen Einblicke liegt daher zum einen auf der Ebene der Kirchenleitung in Rom, zum anderen - als Fallbeispiel - auf der Katholischen Kirche in Österreich sowie der Erzdiözese Wien.

\section{Der sozioreligiöse Kontext (Perspektive a)}

Die Katholische Kirche findet sich heute in einer Welt, in der religiöse Diversität als Faktum anzuerkennen ist. Die Diversifizierung der globalen religiösen Landschaft wird zudem durch Säkularisierung und Migration dynamisiert. Dieser Kontext bildet die Ausgangssituation aller - auch theologischer - Überlegungen zum Umgang mit religiöser Diversität. 


\subsection{Die globale religiöse Landschaft}

Die Forschung des PEW-Research-Center's Forum on Religion und Public Life ermöglicht eine Skizzierung der zeitgenössischen „global religious landscape“2. Von einem Verschwinden der Religion kann keine Rede sein. So wussten sich 2012 5,8 Milliarden Menschen einer Religionsgemeinschaft zugehörig, das sind 83,7 \% der Weltbevölkerung. Nur 16,3 \% der Weltbevölkerung gehören keiner religiösen Gemeinschaft an; die Mehrheit dieser Gruppe lebt im asiatisch-pazifischen Raum. Europa ist nicht so religionsfern wie oft behauptet wird und bildet dort, wo Kirchen und Glauben tatsächlich stark reduziert sind (Tschechien, Ostdeutschland, Frankreich, die skandinavischen Länder), die globale Ausnahme.

Die religiös Zugehörigen teilen sich nach dieser Studie auf wie folgt: 0,2 \% gehören dem Judentum, 31,5 \% dem Christentum, 23,2 \% dem Islam, 7,1 \% dem Buddhismus, $15 \%$ dem Hinduismus, 5,9 \% traditionellen „Folk Religions“ und $0,8 \%$,anderen religiösen Gruppierungen“ an. Keine der hier benannten Religionen ist ein monolithischer Block; jede kennt intern plurale Interpretationen und Praxisformen.

2014 widmete sich das PEW-Research-Center der Messung globaler religiöser Diversität. Der dafür entwickelte Diversity-Index orientiert sich an folgenden Parametern: ${ }^{3}$ 1) an der Quantität der verschiedenen religiösen Gruppen in einer Gesellschaft 2) am sichtbaren, rechtlich gesicherten Einfluss der Minoritätengruppen in der Zivilgesellschaft, 3) am Grad der Dominanz der religiösen Gruppen. Auf einer Skala von 1 bis 10 bedeutet ein hoher Index hohe religiöse Diversität. Kein einziges Land in Europa und Nord-Amerika sowie in der Region des mittleren Ostens bzw. des arabischen Afrika hat einen hohen RDI. Europa hat einen moderaten RDI. ${ }^{4}$ Nur im asiatisch-pazifischen Raum lassen sich Länder finden, deren RDI höher als 7 ist. ${ }^{5}$ Die (gesellschafts)politische und mediale Aufregung angesichts religiöser Diversität in Europa wirkt im globalen Vergleich sehr befremdlich und zeigt, wie ungeübt das heutige Europa im Umgang mit religiöser "Andersheit“ ist. ${ }^{6}$ Der RDI verdeutlicht auch, wie eng das Zusammenleben religiös verschiedener Gruppen mit (Religions)Recht, öffentlicher Anerkennung sowie Macht und Gerechtigkeit zusammenhängt.

2 PEW-Research-Center's Forum on Religion and Public Life 2012a.

3 PEW-Research-Center's Forum on Religion and Public Life 2014.

4 Österreichs RDI $=3.8$, Deutschlands RDI $=5.3$, RDI des Vatikan $=0.0$.

5 In Singapur, Taiwan, Vietnam, Süd-Korea, China und Hongkong sowie in Surinam (Lateinamerika).

6 Eine Ausnahme mögen hier die ehemaligen Vielvölkerstaaten des British Empire und der Habsburger Monarchie bilden, siehe Reiss in diesem Heft. 
Die jüngste Studie des PEW-Research-Center prognostiziert für 2050 folgende Entwicklungen: ${ }^{7}$ ChristInnen werden zwar die größte Gruppe bleiben, aber der Islam wird die am schnellsten wachsende Religion sein. Wenn die aktuellen demographischen Entwicklungen konstant bleiben, wird die Zahl der MuslimInnen weltweit die der ChristInnen eingeholt haben. Die Gruppe der AtheistInnen und AgnostikerInnen und anderer Nicht-Zugehöriger wird zwar in einzelnen Ländern - wie z.B. in Frankreich oder den USA - wachsen, aber weltweit kleiner werden. 2050 wird es der Studie zufolge mehr HinduistInnen und JüdInnen geben als gegenwärtig. In Europe werden MuslimInnen ca. 10 \% der Bevölkerung ausmachen. Indien wird das Land mit der größten muslimischen Bevölkerung weltweit sein. Vier von zehn ChristInnen weltweit werden in Afrika südlich der Sahara leben.

\subsection{Faktoren der Diversifizierung}

Religion befindet sich weltweit in massiven Veränderungsprozessen, deren Folgen heute noch gar nicht abschätzbar sind: sozial, kulturell, politisch, religiös und spirituell. Die die medialen und politischen Diskurse in Europa aktuell dominierenden religionsfundamentalistischen Phänomene können auch als aggressive Reaktion auf die damit verbundenen Orientierungskrisen gedeutet werden. Zeitgleich bildet sich aber auch „derzeit so etwas wie ein globales Bewusstsein vieler miteinander mehr oder weniger vernetzter Spiritualitäten heraus“" , wodurch ein offenes Weltsystem der Religionen entsteht. Unter dem Label „Spiritualität“ entsteht ein neues Paradigma der semantischen, pragmatischen sowie strukturellen Transformationsprozesse von Religion.

Zwei Faktoren seien genannt, die die Diversifizierung von Religion beschleunigen:

1. Der globale Prozess der Säkularisierung ${ }^{9}$ führt weltweit zu heterogenen gesellschaftlichen Entwicklungen und damit verbundenen religionspolitischen und religionsrechtlichen Lösungen. ${ }^{10}$ Säkularisierung kann Religion als öffentliche oder politische Größe in den „,privaten“ Hintergrund verdrängen (wie in Frankreich), aber auch mit Religion im öffentlichen und politischen

7 PEW-Research-Center's Forum on Religion and Public Life 2015.

8 Baier 2006, S. 13.

9 Vgl. zum Folgenden Buß / Luber 2013.

10 Marian Burchardt und Monika Wohlrab-Sahr sprechen sogar von „Multiplen Säkularitäten“ und unterscheiden zwischen Säkularisierung a) im Dienst individueller Freiheit (z. B. in den USA), b) im Dienst der Befriedung religiöser Differenz (z. B. in Indien, Niederlande), c) im Dienst des gesellschaftlichen Fortschritts und nationaler Einheit (in Frankreich, in der kemalistischen Türkei) sowie d) im Dienst der Autonomie gesellschaftlicher Teilbereiche. Burchardt / Monika Wohlrab-Sahr 2013, S. 35-52. 
Raum koexistieren (wie in den USA, Indien). Säkularisierung kann religionsproduktiv wirken, wie das Entstehen alternativer, spiritueller Bewegungen und Gruppen in Europa ${ }^{11}$ zeigt.

2. Migration forciert Pluralisierungs- und Diversifizierungsprozesse im Feld der Religionen und in den sozioreligiösen Landschaften: Religionsgemeinschaften pluralisieren sich in ihrem Inneren, die religiösen Zusammensetzungen verändern sich und die damit verbundene religiöse Diversität nimmt national und lokal zu. ${ }^{12}$ Die Mehrheit der internationalen MigrantInnen weltweit sind ChristInnen und nicht, wie medial immer wieder der Anschein erweckt wird, MuslimInnen: $49 \%$ der internationalen MigrantInnen weltweit sind ChristInnen, 27 \% MuslimInnen. ${ }^{13}$ Auch in der Europäischen Union verhält es sich so: $56 \%$ der MigrantInnen in der EU sind ChristInnen, $27 \%$ MuslimInnen. Den größten Anteil an MigrantInnen hat das Judentum (25\%), es folgen ChristInnen (5\%) und MuslimInnen (4\%). In manchen europäischen Großstädten wie Hamburg und Rotterdam stammt die Mehrheit der Christen aus einem nicht-europäischen Land. ${ }^{14}$ Man spricht von einer „Enteuropäisierung des europäischen Christentums ${ }^{\text {"15.15 }}$. Für JüdInnen verliert Europa seine jahrhundertealte zentrale Bedeutung ${ }^{16}$; der Schwerpunkt verlagert sich in den asiatischen Raum. Ereignisse wie die antisemitischen Ausbrüche während des Gaza-Konflikts im Sommer 2014 oder die Angriffe auf jüdische Einrichtungen im Kontext des Anschlags auf „Charlie Hebdo“ 2015 veranlassen immer wieder JüdInnen Europa zu verlassen.

Migration verändert weiters die Qualität der jeweiligen Mehrheitsreligion: Die neuen Mitglieder bringen neue Praxisformen und Themen mit und können so die autochthone Religionsgemeinschaft beleben. Neue, „fremdartige“ Ideen durch Mission und Konvertiten können zu Innovationen führen und neue Gemeinschaften entstehen lassen. ${ }^{17}$

11 Z. B. Identity Foundation 2006 (für Deutschland); Höllinger 2012 (für Österreich); Stolz / Könemann u. a. 2014 (für die Schweiz).

12 Am Beispiel Wien: Anne Goujon u. a. 2014.

13 PEW-Research-Center's Forum on Religion and Public Life 2012b.

14 Vgl. Bünker 2011, S. 85.

15 Collet 2010, S. 242.

16 Bodenheimer 2014, S. 389-396.

17 Martikainen 2015. 
Regina Polak

\subsection{Fallbeispiel 1: Zum Umgang mit religiöser Diversität von KatholikInnen in Österreich}

Ein anerkennender Umgang mit religiöser Diversität ist freilich in der Katholischen Kirche alles andere als selbstverständlich. Dies sei am Beispiel Österreich gezeigt. In ihren Einstellungen unterscheiden sich katholische Gläubige nicht signifikant vom Rest der österreichischen Bevölkerung. Diese zeichnet sich laut der Europäischen Wertestudie 2008 durch ausgeprägte Schwierigkeiten im Umgang mit Menschen aus, die als „anders“ gelten, allem voran mit MigrantInnen und Minoritäten. ${ }^{18}$ Diese Einstellungen wurden auch in einer Online-Befragung ${ }^{19}$ erkennbar, die das Zukunftsforum $3000^{20}$ der Katholischen Kirche 2013 in Österreich in Auftrag gegeben hat.

Diese Umfrage fragte nach einem „gedeihlichen Miteinander“ im Zusammenleben mit Menschen verschiedener Kulturen, Überzeugungen und Haltungen. Die Ergebnisse zeigten gravierende Bruchstellen der Befragten im Leben mit sogenannten „Ausländern“. Aussagen wie folgende dominierten die Stellungnahmen der katholischen TeilnehmerInnen:

„Dieses eigentlich offensichtliche Bewusstsein - nämlich dass ich mich an die herrschende Kultur anpassen muss wenn ich in der Fremde bin - ist, so scheint's mir, in unseren Breiten zusammen mit dem Bewusstsein für die Koordinaten unserer eigenen Kultur verlorengegangen. Wir können nicht von anderen erwarten, sich an etwas anzupassen, wenn wir selber keine klare Identität haben. Genau da drückt für mich der Schuh: wenn ,Zusammenleben“ nur darin besteht, dass wahllos zusammengewürfelte Leute in ihren angestammten kulturellen Systemen wie in Luftblasen nebeneinander her leben und dabei schlecht oder recht einen improvisierten Modus Vivendi erfinden, dann geht das kurzfristig gut, ist aber letztendlich steril. Deshalb wäre es eine dringende Aufgabe der öffentlichen Autoritäten, eine klare Position zur Frage einer Leitkultur zu finden, die dem christlichen, genauer gesagt katholischen, Erbe der österreichischen Regionen entspricht. Solange der Staat sich hier nicht klar positioniert, wird kein kulturell fruchtbares ,Zusammenleben“ möglich sein. ${ }^{\text {“21 }}$

Viele der Befragten sprechen sich für eine starke österreichisch-christliche Leitkultur aus und erwarten von den zugewanderten MitbürgerInnen Anpassung an die Mehrheit und deren Werte.

Vereinzelt finden sich auch Stellungnahmen, die Vielfalt als Bereicherung erkennen und sich für interkulturelles Zusammenleben, gemeinsame Feste und Feiern sowie eine zuwanderungsfreundliche Migrationspolitik im Verein mit

18 Rosenberger / Seeber 2011, S. 165-189.

19 Zukunftsforum der Katholischen Kirche in Österreich 2013.

20 Katholische Aktion Österreich 2015a.

21 Ibid., Datensatz „Offene Fragen“. Sprachliche und Interpunktionsfehler wurden belassen. 
Kampf gegen Rassismus und Islamophobie aussprechen. Aber selbst in diesen Aussagen steht einer „Wir“-Gruppe von ÖsterreicherInnen die Gruppe der „Anderen“ gegenüber: MigrantInnen, vor allem Muslime, werden auch in diesen Texten nicht als MitbürgerInnen anerkannt:

„Andere Kulturen und Religionen werden zu oft als Bedrohung angesehen, anstatt als Bereicherung. Es ist wichtig, seine eigene Kultur und die anderen Kulturen zu kennen, und Kontakt miteinander zu haben, z.B. Feste zu feiern. Feiertage: Warum gibt es in Österreich fast nur katholische Feiertage? Ich wäre dafür muslimische und jüdische Feiertage auf Kosten der katholischen Feiertage einzuführen. Die Feiertage sollten dann genützt werden, um zusammen zu feiern!‘‘22

Die Umfrage ist weder für Österreich noch für KatholikInnen repräsentativ, lässt aber erahnen, dass die österreichische Kirche in ihrem Inneren massive Schwierigkeiten im Umgang mit (nicht nur) religiöser Diversität hat.

\subsection{Fallbeispiel 2: ,Wir sind keine Indianer!“623 - Erfahrungen einer kroatisch-katholischen Gemeinde in Wien}

Das folgende Beispiel zeigt, wie schwierig das Zusammenleben selbst mit langansässigen Gläubigen einer anderssprachigen Gemeinde in einer katholischen Diözese sein kann.

Die religiöse Struktur der Stadt Wien hat sich in den vergangenen Jahrzehnten gravierend verändert: So waren 1971 satte 78,6 \% der Bevölkerung katholisch, 10,3 \% ohne Bekenntnis, 7,8 \% protestantisch, 1,8 \% gehörten zu ,anderen Bekenntnisgemeinschaften“, $1,1 \%$ waren orthodox und 0,4 \% muslimisch. 40 Jahre später, 2011, sieht die Verteilung aus wie folgt: 41,3\% katholisch, 31,6\% ohne Bekenntnis, 11,6\% muslimisch, 8,4 \% orthodox, 4,2\% protestantisch, 2,9\% zählen zu ,,anderen Bekenntnisgemeinschaften“ ${ }^{24}$ Säkularisierung und Migration transformieren auch das wachsende Wien. Die genauen Zahlen der einer Religionsgemeinschaft zugehörigen Menschen mit sogenanntem „Migrationshintergrund" sind zwar nicht bekannt, aber dass Wien auch in religiöser Hinsicht eine Migrationsstadt ist, ist evident. ${ }^{25}$ So haben $15-20 \%$ der Katholikinnen und Katholiken (ca. 100.000-150.000 Personen) in Wien einen migrantischen Hintergrund. Davon stammen zwei Drittel aus Europa und ein Drittel aus Asien, Afrika

22 Ibid., Sprachliche und Interpunktionsfehler wurden belassen.

23 Polak 2015.

24 Anne Goujon u.a. 2014.

25 Wien ist eine Migrationsstadt: 49 \% der ca. 1700000 Personen, die in Wien leben, haben einen sogenannten Migrationshintergrund, d.h. mindestens einer der beiden Elternteile ist nicht in Österreich geboren, vgl. Integrations- und Diversitätsmonitor der Stadt Wien 2009-2011. 
und Lateinamerika. Die katholischen MigrantInnen sind in 24 anderssprachigen Gemeinden organisiert. ${ }^{26}$ Diese migrationsbeschleunigte innere Diversifizierung wird aber weder von der Ortskirche in Wien noch von Forschung und Politik angemessen zur Kenntnis genommen.

Die katholische „Immer-Noch“-Mehrheitskirche in Wien ${ }^{27}$ ist ihren anderssprachigen „Brüdern und Schwestern im Glauben“ gegenüber erstaunlich blind. Wohl gibt es immer wieder heftige Auseinandersetzungen rund um und mit anderssprachigen Priestern. ${ }^{28}$ Die anderssprachigen Gemeinden jedoch wurden bis vor kurzem bestenfalls als Gäste, nicht aber als selbstverständlicher Teil der Ortskirche von Wien wahrgenommen. Diese Erfahrung des Nicht-Wahrgenommen-Werdens spiegelt sich wieder in der Abwesenheit von VertreterInnen in den diözesanen Gremien, Strukturen und Veranstaltungen. Die Erfahrungen der kroatischen Gemeinde in Wien - die größte der anderssprachigen Sprachgruppen in Wien - sind vermutlich repräsentativ für die „Migrations- und Diversitätsblindheit" der Ortskirche von Wien.

2012 habe ich in dieser Gemeinde eine qualitative Studie durchgeführt. ${ }^{29}$ Die Befragten meiner Studie - Priester wie Laien - erzählten vom Mangel an Erfahrung von Zugehörigkeit zur Ortskirche, von dem zu wenig erlebten Gefühl „Teil der österreichischen Kirche zu sein“"30. So erfuhr die kroatische Niederlassung in Wien (die „Mission““31) zu Beginn - in den 1960er-Jahren im Zuge der Zuwanderung von Gastarbeitern - von Seiten der Diözese kaum Unterstützung. Seit 1969 hat die Gemeinde eine eigene Kirche, seit 1973 den Status einer Personalpfarre. Der Kontakt mit österreichischen Pfarren und Priestern wird aber nach wie vor als ungenügend wahrgenommen. Die Befragten erzählten, dass sie von Wiener Gläubigen als Ultrakonservative und Traditionalisten wahrgenommen werden. Mehrfach wurde die Ignoranz der Einheimischen erwähnt.

Besonders beklagt wurde in den Gesprächen der sogenannte „Sonntag der Völker“, ein Sonntag im September, an dem die Wiener Erzdiözese mit ihren anderssprachigen Gemeinden Gottesdienst feiert - ein Gottesdienst, bei dem die

\section{Anderssprachige Gemeinden in Wien 2015.}

27 Die Erzdiözese Wien umfasst auch große Teile von Niederösterreich. In diesem Beitrag ist mit Erzdiözese Wien stets nur die Stadt Wien gemeint.

28 Die Pastoral in Wien wird allerdings seit dem 19. Jahrhundert zu einem großen Teil von ausländischen Priestern verantwortet. Das Phänomen ist also nicht neu. Die Erzdiözese Wien hat mittlerweile eine „Interkulturelle Akademie für Priester (IKAP)“ eingerichtet, in der ausländische Priester besser für den Dienst in Wien vorbereitet werden sollen.

29 Polak 2015; Polak 2013.

30 Die unter Anführungszeichen gesetzten Zitate sind Originalzitate aus der Studie.

31 Hrvatska Katholička Misija Beč 2015. Missionen sind von der Katholischen Kirche eingerichtete diözesane Gemeindeniederlassungen - Sprach- und Ritusgemeinden - für MigrantInnen. 
ansässigen Gläubigen alljährlich durch Absenz glänzen. „In Wien hat man den ersten schwarzen Mann ausgestopft - so kommen wir uns beim ,Sonntag der Völker' vor. Wir kochen zuhause nicht mit offenem Feuer", meinte ein Interviewpartner. Auch die vollständige Absenz der Ortskirche beim ersten Wiesenfest im Mai 2012, bei dem die anderssprachigen Gemeinden im Sigmund-FreudPark vor der Votivkirche in Wien zu einem Fest mit zahlreichen Darbietungen einluden, wurde als „Antwort“ interpretiert: Die Ortskirche hat an anderssprachigen Gemeinden kein Interesse. Diese Erfahrungen führen zu „Schmerz, auf einer seelischen Ebene, der schwer zu verkraften ist". Einer der befragten Priester strich heraus, ,dass die Kroaten durchaus bereit sind, sich anzupassen und das auch tun, aber dass auch sie verletzlich sind und beachtet werden wollen". Diese Beachtung wünschen sich die Befragten nicht nur für ihre „exotischen“ kulinarischen oder tänzerischen Beiträge bei Festen und Feiern, sondern auch in pastoralen Fragen: „Nehmen Sie uns nicht wie Indianer, wir sollen uns Trachten anziehen, ein bisschen spielen und dann gehen wir nach Hause. Wir haben etwas zu fragen, auch etwas zu sagen." So formuliert ein Priester einer kroatischen Gemeinde in der Erzdiözese Wien seine Erfahrungen mit einem autochthonen Pfarrer, der nicht erlaubte, dass die kroatische Gemeinde einen inhaltlichen Teil zu einer Veranstaltung zu Familienpastoral beiträgt.

Die Befragten erzählten, dass einzelne Gemeindemitglieder individuelle Lösungen der Integration praktizieren. Sie besuchen Angebote in autochthonen Pfarren, zu denen sie eingeladen werden. Aber dies wird oft als Überlastung erlebt und führt auch zu Gefühlen der Zerrissenheit. Viele reagieren auf das Desinteresse der Ortskirche mit Rückzug und sind sich der Segregationsprozesse durchaus bewusst, meinte der Pfarrer der Gemeinde: „Wir wissen auch, dass es eine gewisse Holschuld von den Migranten gibt. Man muss auch sagen: jetzt, o.k., was bin ich bereit auch selber zu geben? Es haben sich aber auch in diesem Vakuum, wo sich keiner interessiert hat, die eigenen Strukturen gebildet, die natürlich dann zu Parallelwelten werden. [...] Und irgendwann einmal sagen die fremdsprachigen Gemeinden: Na ja, wir machen eh das, was wir wollen.“

Meine Studie zeigt, dass die kroatische Gemeinde nicht angemessen an den innerhalb der diözesanen Strukturen laufenden Kommunikations- und Entscheidungsprozessen teilnehmen kann. Ob und wie die derzeit laufenden Strukturreformen in der Erzdiözese, bei der die nicht mehr finanzierbare Vielzahl der Pfarren durch Zusammenlegungen zum Modell „Pfarre neu“ reduziert werden soll, als Möglichkeit wahrgenommen wird, anderssprachigen Gemeinden mehr Partizipation in der Ortskirche zu eröffnen, lässt sich derzeit noch nicht sagen. Informelle Gespräche liefern ein widersprüchliches, konflikthaftes Bild: Während sich die Kirchenleitung mehr Integration wünscht, sind Vertreter der anderssprachigen Gemeinden besorgt um die Aufrechterhaltung ihrer muttersprachlichen Gemeinden. Diese Konflikte lassen zumindest ahnen, dass die anderssprachigen Gemeinden nicht mehr unsichtbar sind. 


\section{Relevanz religiöser Diversifizierungsprozesse (Perspektive b)}

Als ,global player“ in der Weltpolitik und als Teil der Zivilgesellschaft trägt die Katholische Kirche mit ihren Ortskirchen universale und lokale Mitverantwortung in einer Welt, in der die Frage nach einem friedlichen und gerechten $\mathrm{Zu}$ sammenleben in (nicht nur) religiöser Diversität zur Überlebensfrage der Menschheit wird. Diese Verantwortung hat auch theologische Ursachen. Die universale Bedeutung des Evangeliums als Botschaft des Heils für alle Menschen und das Selbstverständnis der Kirche als Zeichen der Einheit verpflichtet zum Dienst der Versöhnung und zum Einsatz für Gerechtigkeit und Frieden zwischen den Völkern in ihrer Verschiedenheit. Vier aktuelle „Zeichen der Zeit“ lassen diese Verantwortung zu einer besonderen Herausforderung werden:

1) Das friedliche Zusammenleben mit dem kulturell „Anderen“ wird weltweit dringlicher durch Migration und Flucht und daher inner- wie außerhalb der Kirche zur Aufgabe der Stunde. Das Lehramt der Katholischen Kirche sieht in der Instruktion „Erga Migrantes Christi“ in den weltweiten Migrationen einen Weg Gottes, die Heilsgeschichte voranzutreiben: „Wir können also das gegenwärtige Migrationsphänomen als ein sehr bedeutsames ,Zeichen der Zeit' betrachten, als eine Herausforderung, die es beim Aufbau einer erneuerten Menschheit und in der Verkündigung des Evangeliums des Friedens zu entdecken und zu schätzen gilt." 32 Migration und die damit verbundenen kulturellen Diversifizierungsprozesse werden als Initiative und Form der Anwesenheit Gottes gelesen, die den Menschen erlaubt, sich für Friede, Gerechtigkeit und Solidarität zu engagieren: „Der Übergang von monokulturellen zu multikulturellen Gesellschaften kann sich so als Zeichen der lebendigen Gegenwart Gottes in der Geschichte und in der Gemeinschaft der Menschen erweisen, da er eine günstige Gelegenheit bietet, den Plan Gottes einer universalen Gemeinschaft zu verwirklichen."33 Das hat praktische Auswirkungen auf die Wahrnehmung und den Umgang mit dem kulturell und religiös „Anderen“: „Die Christen sind daher aufgerufen, zusätzlich zum Geist der Toleranz - die außer einer großartigen politischen und kulturellen auch eine religiöse Errungenschaft darstellt - die Achtung vor der Identität des Anderen $\mathrm{zu}$ bezeugen und $\mathrm{zu}$ praktizieren, indem sie, wo es möglich und angebracht ist, Wege des Teilens mit Menschen unterschiedlicher Herkunft und Kultur beschreiten." 34

2) Untrennbar damit verbunden ist der Einsatz für (internationale) Gerechtigkeit, da die internationalen Migrationen maßgeblich durch „die Zunahme der Ungleichheit zwischen der Nord- und Südhemisphäre, die Existenz von protektionistischen Schranken im internationalen Handel, die den aufstrebenden

32 Erga Migrantes 14.

33 Erga Migrantes 9.

34 Ibid. 
Ländern nicht erlauben, die eigenen Produkte zu Wettbewerbsbedingungen auf den Märkten der westlichen Länder anzubieten, und schließlich die Vermehrung von Konflikten und Bürgerkriegen“"35 verursacht sind und aufgrund eines ungerechten Wirtschaftssystems auch zukünftig noch zunehmen werden. Merkmal einer gerechten Welt ist aus einer bibeltheologischen Perspektive, der sich das kirchliche Lehramt bezüglich Migration und Flucht verpflichtet weiß, der Umgang mit den sogenannten „Fremden“36. Die Lebensqualität von Minoritäten und MigrantInnen, ungeachtet ihrer religiösen Zugehörigkeit, ist der „Qualitätsmaßstab“ für die Humanität und Gerechtigkeit einer Gesellschaft.

3) Die globalen kriegerischen und terroristischen Auseinandersetzungen haben ihre Ursachen eher in sozialen, ökonomischen und politischen Unrechtsverhältnissen als in Religion. Im Kontext von Armut, Ausbeutung und Sklaverei werden Religionen in verschiedener Weise allerdings zu „politischen" Akteuren. Sie forcieren ihren Einsatz für Gerechtigkeit und Solidarität, können Religion als Opiat für die Armen offerieren oder ihren fundamentalistischen Terror gegen die (vermeintlichen) Verursacher von Not und Armut richten. In Europa, das diese komplexen Zusammenhänge gerne ausblendet, um die eigene Verstrickungen in Menschen- und Waffenhandel und neokolonialistische Ausbeutungsverhältnisse zu verschleiern, werden die Ursachen von Krieg und Terror nicht selten „,religionisiert“. Sie werden monodimensional „der Religion“ als solcher zugeschrieben: „Der“ Islam ist dann verantwortlich für die Schwierigkeiten in der europäischen Bildungslandschaft, „,die“ JüdInnen verhindern Frieden im Nahen Osten, „die“ Religion ist schuld an allen Formen terroristischer Gewalt. So lässt sich in Europa eine beunruhigende Zunahme islamophober ${ }^{37}$ und antisemitischer Einstellungen ${ }^{38}$ beobachten, die im Verein mit Rassismus, Fremdenhass und anderen Arten des Menschenhasses zu einem erschreckenden Einzug rechtspopulistischer politischer Einstellungen in die Mitte der Gesellschaft führen. Die Kirche ist verpflichtet, gegen jede Art von Menschenhass aufzutreten.

\section{Erga Migrantes 4.}

36 Erga Migrantes begründet seine Sicht auf Migration biblisch, indem es in Abschnitt 14 u. a. an den Auszug des Stammvaters Abraham in ein fremdes Land erinnert; das Gebot Lev 19,34 in Erinnerung ruft, demzufolge der Fremde zu lieben ist, wie man sich selbst liebt. Ebenso wird in EM 16 der Ursprung der jungen Kirche als Fremde in der Welt zitiert. Die Liebe zu den MigrantInnen wird christologisch begründet: „Die Kirche hat in den Migranten immer das Bild Christi gesehen, der gesagt hat: ,Ich war fremd und obdachlos, und ihr habt mich aufgenommen' (Mt 25, 35).

37 Vgl. Agentur der Europäischen Union für Grundrechte 2006. Vgl. für Deutschland z. B. Hafez / Schmidt 2015.

38 Vgl. Agentur der Europäischen Union für Grundrechte 2014. 
4) Die historische Verantwortung nimmt die Katholische Kirche ebenso in die Pflicht. Die Europäische Wertestudie konnte 2008-2010 einen europaweit signifikanten Einfluss normativ-religiöser Einstellungen auf das gesamte Wertesystem feststellen: Wer sich selbst als religiös versteht, hat mit signifikant größerer Wahrscheinlichkeit höhere Zustimmungswerte zum Wert von Ehe und Familie sowie zur Bedeutung von Erwerbsarbeit, betont stärker Solidarität, hat aber auch höhere Werte bei Autoritarismus, Homophobie und Fremdenfeindlichkeit. ${ }^{39}$ In diesen Ergebnissen, die vor allem im orthodoxen Osten Europas deutlich ausgeprägt sind, spiegeln sich weniger die Folgen des christlichen Glaubens als vielmehr die Geschichte der Kirche(n) wider, die sich und den christlichen Glauben in enger Kooperation mit Staat und Politik in den Dienst von Ordnungspolitik gestellt hat bzw. haben. Dieses Erbe belastet in Europa die Glaubwürdigkeit der Kirche im Einsatz für Anerkennung religiöser Diversität.

\section{Theologische Hermeneutik religiöser Diversität (Perspektive c)}

Für die Katholische Kirche ist die Frage nach Wahrnehmung und Umgang mit religiöser Diversität nicht bloß eine pragmatische Herausforderung. Ohne eine theologische Interpretation der sozioreligiösen Diversität der Gegenwart kann sie ihre Verantwortung nicht angemessen wahrnehmen.

\subsection{Belastete Tradition: Partizipation an der Wahrheit der Katholischen Kirche als Lösung?}

Wer die religiös „Anderen“ aus der Sicht des Glaubens sind und wie mit ihnen zu leben ist, ist theologisch keine neue Frage. Ebenso zentral war immer auch die Frage, wie man mit der kircheninternen religiösen Verschiedenheit umgehen soll. Historisch hat die Katholische Kirche verschiedene theologische „Lösungen“ für diese Fragen gefunden, welche freilich zu Diskriminierung, Unterdrückung, Exklusion, Verfolgung, Vertreibung und nicht zuletzt Ermordung der religiös „Anderen“ - von „Ketzern“ und Häretikern, Protestanten, Atheisten und allem voran Juden - geführt haben. Sie sind durch die historischen Folgen desavouiert. Die dogmatische Lehre „Extra ecclesiam nulla salus“ - außerhalb der Kirche kein Heil - hat jedenfalls Millionen Menschen das Leben gekostet, und es steht außer Zweifel, dass der jahrhundertelange „christliche“ Antijudaismus auch die Shoa maßgeblich vorbereitet hat. Auch intern ist es den ChristInnen nicht geglückt, die kirchliche Diversität friedlich zu regeln, wie das Schisma mit der orthodoxen

39 Arts / Halman 2001, S. 86 f. 
Kirche, die Konfessionskriege der Neuzeit, Reformation und gewaltsame Rekatholisierung sowie die bis heute existierenden Kirchentrennungen deutlich zeigen.

Mit dem Zweiten Vatikanischen Konzil (1962-1965) hat die Katholische Kirche neue Wege beschritten. Die „Erklärung Dignitatis Humanae zur Religionsfreiheit“" (1965) sichert jedem Menschen das Recht auf religiöse Freiheit zu. ${ }^{40}$ Die „Erklärung Nostra Aetate über das Verhältnis der Kirche zu den nichtchristlichen Religionen" weiß die Kirche im Judentum verwurzelt und anerkennt den geistlichen Bund mit „dem Stamme Abrahams" ${ }^{\text {“41 }}$, spricht von der „Hochachtung ““42 der Kirche für Muslime und „lehnt nichts von alledem ab, was in diesen Religionen [in Hinduismus und Buddhismus, RP] wahr und heilig ist. “43 Sie gesteht ein, dass auch andere Religionen „den Strahl jener Wahrheit erkennen lassen, die alle Menschen erleuchtet“"44. Schließlich gehört gemäß dem „Dekret Unitatis Redintegratio über den Ökumenismus“ die Wiederherstellung der Einheit aller ChristInnen zu den „Hauptaufgaben“ des Konzils wie auch der Gläubigen. Denn die „Spaltung widerspricht [...] ganz offenbar dem Willen Christi, sie ist ein Ärgernis für die Welt und ein Schaden für die heilige Sache der Verkündigung des Evangeliums vor allen Geschöpfen.“45

Auf den Wahrheitsanspruch der Katholischen Kirche wird dabei freilich nicht verzichtet. Die Katholische Kirche stellt sich immer noch in das Zentrum der Heilsgeschichte. Alle anderen christlichen Kirchen und die anderen Religionen sind ihr im Modus der Teilhabe konzentrisch zugeordnet. Der dogmatischen Frage, ob dieses Partizipationsmodell für die anstehenden Herausforderungen taugt, werde ich hier nicht weiter nachgehen. Stattdessen möchte ich drei Modelle aus der Tradition in Erinnerung rufen, die die Weiterarbeit an einer gegenwartstauglichen Theologie der Diversität unterstützen könnten. Sie antworten alle auf die Frage: Was bedeutet die (religiöse) Diversität der Menschen aus der Sicht des Glaubens?

\footnotetext{
40 Dignitatis Humanae 2.

41 Nostra Aetate 4.

42 Nostra Aetate 3.

43 Nostra Aetate 2.

44 Ibid.

45 Unitatis Redintegratio 1.
} 


\subsection{Der Noah-Bund: Anerkennung von Diversität ${ }^{46}$}

Mit dem Text aus Genesis 10 reagieren die Verfasser auf die Situation ihres Volkes Israel im Exil. Israel sieht sich mit anderen kulturellen und religiösen Traditionen konfrontiert: Warum will Gott die Menschheit als eine Vielfalt aus verschiedenen Völkern und Menschen?

Die Antwort: Gott hat mit Noah einen Bund geschlossen, demzufolge er die Menschheit nie wieder vernichten wird. Alle Völker dieser Welt sind Nachkommen des Noah und seiner Söhne. Sie bilden eine Art „Völker-Familie“.

Zur Erinnerung: Noch keine zehn Generationen sind vergangen, da reut Gott, was er geschaffen hat (Gen 6,5). Er sieht, dass ,auf der Erde die Schlechtigkeit des Menschen zunahm und alles Sinnen und Trachten seines Herzens immer nur böse war" (Gen 6,5). Seine Schöpfung ist durch die Menschen ,voller Gewalttat" (Gen 6,13). Der Schöpfer zerstört seine Schöpfung mittels einer Flut (Gen 7). Der Text soll für Israel eine bleibende Selbstwarnung sein.

Die zweite, nachsintflutliche Schöpfung folgt veränderten, rigideren Regeln. Nach der Flut spricht Gott erneut einen Fruchtbarkeitssegen (Gen 9,1). Aber diesmal verbindet sich dieser mit einer Drohung. Wieder wird alles, was sich auf Erden regt, den Menschen übergeben. Die Tiere jedoch leben künftig in Furcht und Schrecken vor dem Menschen. Die Schöpfung hat ihre Unschuld verloren; sie ist eine andere geworden. Eine absolute Grundregel gibt Gott dieser neuen Menschheit: Das Töten von Menschen ist und bleibt Tabu. Es gilt als Brudermord und für jeden, dessen Blut vergossen wird, wird Rechenschaft verlangt. Sodann verpflichtet sich Gott auf den Erhalt der Schöpfung: Nie wieder sollen alle Wesen ausgerottet werden, nie wieder soll eine Flut die Erde vernichten (Gen 9,8). Gott schließt einen Bund, nicht nur mit den Menschen: den noachidischen Bund Gottes mit der gesamten Schöpfung.

Diese Erzählung ist Ausdruck des universalen Denkens Israels. Israel bindet seine Geschichte in eine umfassende Schöpfungsgeschichte. Der Bund Gottes mit allen Menschen und der ganzen Schöpfung bildet Rahmen und Basis aller weiteren Ereignisse und Bundesschlüsse.

Die Söhne Noahs - Jafet, Ham und Sem - sind die Vorfahren der verschiedenen Völker. Sie sind im Ursprung über Noah miteinander verbunden. Nicht nur alle Menschen (über Adam), auch alle Völker sind miteinander verwandt. Die Vielfalt der Völker ${ }^{47}$ ist gewollt. Alle Völker sind gleich und haben einen gemeinsamen Ursprung. Die theologische Pointe dieser Erzählung besteht in der Aussage über die Einheit des Menschengeschlechtes. Segen ist auch den Nicht-Erwählten zugesagt.

46 Abschnitt 4.2 folgt Kuschel 2013, S. 234-249.

47 Der Begriff des Volkes ist hier nicht rassisch-völkisch, kulturalistisch-national, sondern geschichtlich-geographisch zu verstehen. Volk bedeutet, dass die Verbindung von Menschengruppen in Raum und Zeit Erinnerungsgemeinschaften stiftet. 
Diese Einheit und Gleichheit wird allerdings sofort wieder gestört. Noah verflucht seinen Enkel Kanaan, den Sohn Hams, und verurteilt ihn zum Knecht Sems. Damit brechen erneut Ungleichheit, Unterdrückung und Gewalt in die Völkerwelt ein.

Eine weitere Spannung wird durch die Auserwählung Israels durch Gott in die Menschheitsgeschichte gebracht. Israel bekommt die Aufgabe, „Licht der Völker" zu sein und die Völker dadurch zu bewegen, Jahwe zu verehren. Damit wird eine neue Denkweise des Verhältnisses zwischen der Partikularität eines Volkes (Israel) und der Universalität der Menschheit eröffnet: Israel lebt nicht wie die Völker, aber auch nicht gegen sie - sondern verschieden. Israels Leben schwankt zwischen den Polen von Partikularität (Auserwähltheit, Differenz, Separation) und Universalität (Verantwortung für die Gesamtmenschheit). Israel ist verpflichtet zu Solidarität mit den Weltvölkern - ein Modell, das in der Antike ohne Analogie ist.

Die Noah-Erzählung eignet sich dazu, die religiös Verschiedenen als „Kinder Noahs “" ${ }^{48}$ zu betrachten. So spielt sie nicht ohne Grund eine zentrale Rolle bei der Frage nach religiöser Diversität bei JüdInnen ${ }^{49}$, ChristInnen und MuslimInnen $^{50}$,

48 Kuschel 2013, S. 304-305.

49 So hat das rabbinische Judentum im Babylonischen Talmud die sogenannten „Noachidischen Gebote“ formuliert, die auch Nicht-Juden, die diese Gebote halten, als „Fromme vor Gott“ bezeichnen. Als eine Art ethischer Minimalverpflichtung umfassen sie das Gebot der Rechtspflege, die Verbote von Götzendienst, Gotteslästerung, Unzucht, Blutvergießen, Raub, sowie des Genusses eines Gliedes vom lebendigen Tier (Grausamkeit gegen Tiere). Theologisch heißt das: Der Fremde, der nicht zum Volk gehört und gehören will, hat einen eigenen theologischen Status: Er wird der geistigreligiöse Nachbar, dem ebenfalls Gottes Gerechtigkeit zugesagt wird. Moses Maimonides wird diesen Begriff, den ger toschav, mit dem Begriff „Gerechter unter den Völkern“ verbinden: „Wer die sieben Gebote übernimmt und gewillt ist, sie zu tun, gehört zu den Frommen der Weltvölker und hat Anteil an der kommenden Welt; dies gilt für jenen, der sie übernimmt und tut, weil Gott in der Tora so geboten und durch unseren Lehrer Moses bekannt gemacht hat, dass die Nachkommen Noahs auf diese Gebote verpflichtet wurden. Wenn jemand hingegen aufgrund der Überzeugung durch den eigenen Verstand tut, ist er kein ger toschav und kein Frommer der Weltvölker und keiner ihrer Weisen. " Moses Maomonides, zitiert nach Kuschel 2013, S. 287.

50 Der Koran bekräftigt die prinzipielle Gleichheit aller Menschen vor Gottes Gesetz. Kein Mensch ist vom Gesetz Gottes ausgenommen (Sure 5,123). Zugleich weiß der Koran um die faktische Zersplitterung der Menschheit, der aber eine „Theo-Logik“ unterstellt wird: Dass Gott verschiedene Völker und Stämme zugelassen hat, hat einen Sinn: Menschen sollen einander kennen lernen und untereinander um das Gute wetteifern. So z. B. Sure 49,13: „Ihr Menschen, wir haben Euch zu Völkern und Stämmen gemacht, damit ihr einander kennt. Der Edelste vor Gott ist der unter Euch, der am gottesfürchtigsten ist.“ Oder Sure 30,22: „Und zu seinen Zeichen gehören die Erschaffung der Himmel und der Erde, die Verschiedenheit eurer Sprache und Farben. Darin 
die sich auf diese Erzählung beziehen. Sie lassen sich alle als „Kinder Noahs“ betrachten und sind aufgrund ihrer „Verwandtschaft" vor Gott verpflichtet, zueinander gerechte und friedliche Beziehungen aufzubauen.

\subsection{Diversität - Erfahrung der Zersplitterung, Schutz der Fremdheit}

Der hebräische Tenach wie das christliche Alte Testament anerkennen Diversität also als von Gott geschaffenes und gewünschtes Faktum, gleichwohl verbindet sich in diesen Texten Diversität auch mit Gewalt und Konflikt. So folgt denn nach der Völkertafel in Genesis 10, die die nach der Sintflut neu entstandene Vielfalt der Stämme und Sippenverbände, deren Heimatregion, Personen und Geschlechterfolgen schildert, in Genesis 11 eine weitere Ätiologie. Diese erklärt Grund, Sinn und Ziel kultureller, religiöser und sprachlicher Diversität mittels der Erzählung vom Turmbau zu Babel (Gen 11).

Der Exeget Jürgen Ebach liest diese Erzählung als differenzierte Bejahung, ja sogar als „Rettung“ der Vielfalt. ${ }^{51}$ Alle Menschen sprechen in Gottes „zweiter Chance “52 für die Schöpfung die gleiche Sprache. Dann aber versuchen sie, diese Einheit abzusichern: Ein Turm soll gemeinsam gebaut werden - damit sie sich „einen Namen machen“ und „nicht mehr über die ganze Erde zerstreuen“ (Gen 11,4). Die Menschen werden zu Funktionen dieses Einheitsprojektes. Die Sprache verkommt auf eine der Organisation und der Befehle: „Auf, formen wir Lehmziegel und brennen wir sie zu Backsteinen!“ (Gen 11,3) Verständigung dient nun nicht mehr dem wechselseitigen Verstehen, sondern dem Schaffen eines gemeinsamen Projektes, dem alle zu dienen haben. In Gen 11 verhindert Gott dieses Projekt: Er zerstört den himmelhoch geplanten Turm, verstreut die Menschen auf der ganzen Erde und verwirrt ihre Sprache. Nun verstehen sie einander nicht mehr. Der biblische Verfasser weiß: Diversität ist nicht nur Bereicherung, sie kann Zerstreuung und „Wirrsal“ (Gen 11,11) nach sich ziehen.

In der Rezeptionsgeschichte wurde Gottes Reaktion immer wieder als „Strafe" gedeutet. Der Text von Ebach tut dies nicht. Nach ihm schützt Gott mit dieser Maßnahme vielmehr die Menschen vor sich selbst: Indem er die Pluralität sichert, versucht er alle Projekte zu blockieren, die eine imperiale Macht schaffen, der alle zu dienen haben. Die Pluralität wird als Diversität erkennbar, die nach Kommunikation zwischen Verschiedenen verlangt und diese in gewissem Sinn sicherstellt. Gott institutionalisiert die Fremdheit zwischen Menschen, um diese vor wechselseitiger Vereinnahmung und Homogenisierung zu schützen. Eine Kommunikation wird nötig, die das Fremde nicht auslöscht. Gen 11 erinnert

sind Zeichen für die Wissenden.“ Verschiedenheit ist „Gesetz Gottes“. Vgl. Kuschel 2013, S. 318.

51 Vgl. Ebach 2008.

52 Kuschel 2013, S. 11. 
daran, dass Einheitsprojekte, die die Verschiedenheit auslöschen, nicht den Menschen dienen und daher nicht Gottes Gefallen finden.

Diversität hat aus bibeltheologischer Perspektive ein Doppelgesicht. Sie ist als Pluralität eine Gabe, ein Geschenk und eine Aufgabe, die durch Kommunikation zu gestalten ist. Ihr Schutz hat Vorrang vor allen Versuchen, Einheit um den Preis der Auslöschung des Verschiedenen, des Fremden herzustellen. Einheit wird verstanden als Kommunikations- und Beziehungsprozess der Teilhabe. Zugleich ist diese Wahrnehmung von Diversität nicht naiv: Diversität kann Menschen voneinander isolieren, Zerstreuung, Konflikt und Gewalt nach sich ziehen. Sie bedarf daher immer auch der Pflege der der Verschiedenheit zugrundeliegenden Einheit des Menschengeschlechts.

Die bibeltheologischen Zugänge ermöglichen der Kirche eine selbstkritische Sicht auf historische tradierte Einheitsvorstellungen sowie eine neue Wahrnehmung von Diversität.

\subsection{Systematisch-theologischer Zugang: Katholizität}

Die Katholische Kirche ist in der Entwicklung ihrer Theologie auch der kirchlichen Tradition verpflichtet. So soll als weiteres Modell eine der vier zentralen nota ecclesiae (Wesensmerkmale der Kirche) - die Katholizität - im Kontext der Frage nach dem Umgang mit Diversität in ihrem Potential erschlossen werden. ${ }^{53}$

Der Begriff „Katholizität“ ist belastet. Zudem wird er seit den Konfessionskriegen im Zuge der Reformation konfessionalistisch verengt verstanden, d.h. mit der römisch-katholischen Kirche identifiziert. Dies ist jedoch eine problematische Reduktion. Auch die orthodoxe und die evangelische Kirche verstehen sich als katholisch.

Daher sei daran erinnert, auf welche Frage dieser Begriff die theologische Antwort war. Frühkirchlich bezeichnet der Begriff ein sowohl theoretisches als auch praktisches Modell, den Zusammenhang zwischen der Fülle des Heils, das von Gott her allen Menschen eröffnet ist, und der Verschiedenheit der Menschen zu denken und zu leben: Wie kann das universale Heil der Vielfalt der Menschen zukommen? Die junge Kirche kommt zunehmend zu der Überzeugung, dass dies nur vermittels ihrer selbst möglich ist.

Der Sache nach wird diese Frage freilich bereits in zahlreichen Texten des Alten Testament reflektiert. Vor allem die Propheten verkünden, dass die Gerechtigkeit Gottes allen Völkern zugesagt ist. In visionären Bildern von einer „Wallfahrt der Völker“ (z. B. Jes 2,2; Jes 60,4-9; Mich 4,1) bringen sie diese Erfahrung zum Ausdruck. Die Völker pilgern zu Jahwe und loben und preisen Gott in der Vielfalt ihrer Sprachen und Kulturen: Sie müssen ihre Identität - auch

53 Dazu ausführlich Polak 2014. 
die religiöse - nicht aufgeben: „Es kommt die Zeit, alle Nationen und Sprachen zu versammeln, dass sie kommen und meine Herrlichkeit sehen.“ (Jes 66,18).

Die Texte des Neuen Testaments stehen in dieser Tradition. Ihre Verfasser glauben, dass die Fülle des Heiles (pleroma) Gottes in Jesus von Nazareth, den sie als den Messias erkennen, irdische Wirklichkeit geworden ist. Die junge Kirche versteht sich als Gemeinschaft jener Gläubigen, die diese „Frohe Botschaft“ allen Menschen in Wort und Tat verkünden soll. In ihr sind Juden und Heiden nicht mehr getrennt, sondern „,in Christus“ miteinander versöhnt (Eph 2,14). In der weiteren theologischen Entwicklung reflektiert die Kirche mittels des Modells der „Katholizität“ folgende Fragen: Wie verhält sich die neu erfahrene Einheit zur Vielfalt und Verschiedenheit der einzelnen Gemeinden, der verschiedenen Kulturen, in die hinein das Evangelium verkündet wird? Wie hängen die Partikularität des Heils, das in der Kirche zur Gänze erfahren werden kann, mit der Universalität des Heils zusammen, das allen zugesagt wird?

Seit jeher versucht die Kirche mithilfe dieses Begriffes also die Frage zu beantworten, wie Vielfalt und Einheit zusammenhängen, woran sich Einheit in der Vielfalt erkennen lässt und wie von daher praktisch zu handeln ist. Der Begriff verhandelt das Verhältnis von Partikularität und Universalität. Freilich erlebte er im Lauf der beiden Jahrtausende seit Christus unzählige Bedeutungswandel. ${ }^{54}$ Was hier so abstrakt-philosophisch klingt, hatte und hat maßgebliche Auswirkungen auf das Verständnis von Macht und Politik im praktischen Umgang mit Diversität, nicht nur in der Kirche, sondern auch in der Gesellschaft. Daher ist folgende Frage nicht nur für die Kirche hochaktuell: Wie ist das Verhältnis von Vielfalt und Einheit, Partikularität und Universalität zu denken und gestalten?

Im Alten wie im Neuen Testament finden sich dazu zahlreiche „Lösungsmodelle“. Diese zeichnen sich durch eine intensive Auseinandersetzung mit der kulturell und religiös anderen Umwelt aus und schildern heftig umstrittene Dynamiken zwischen Rezeption und Abgrenzung. Im Laufe der Geschichte der Kirche wird die Sicherung von Einheit und Universalität freilich zunehmend ausschließlich an sichtbaren kirchlichen Institutionen festgemacht: in der Katholischen Kirche an deren globaler Universalität, dem Festhalten an der kirchlichen Autorität des Bischofsamtes und der Rechtgläubigkeit gegenüber der kirchlichen Lehre. Katholisch sein bedeutet spätestens seit der Konfessionalisierung dieses Begriffes fortan eine universale Gemeinschaft mit einem gemeinsamen Glaubensbekenntnis zu sein, die unter der Leitung von Bischöfen und dem Papst gemeinsam die Sakramente feiert.

Die Katholische Kirche hat erst im Zweiten Vatikanischen Konzil wieder zu der Erkenntnis gefunden, dass dieses Modell inner- wie außerkirchlich zu kurz greift. Die Totalidentifikation ,des“ Heils mit einer einzigen Kirche und deren symbolischen und institutionalisierten Ausdrucksformen ist auch aus der Sicht der Katholischen Kirche mittlerweile eine unhaltbare Verkürzung. Auch inner-

54 Polak 2014. 
kirchlich wurde das Verhältnis zwischen den partikulären Ortskirchen und der universalen Kirche reformuliert. Die Ortskirchen sind weder „Filialen“ noch „Umsetzungsorte“ der Universalkirche. Die Universalkirche ist auch nicht die Summe ihrer Teilkirchen. Vielmehr ist in jeder Ortskirche die ganze Kirche präsent. Für die Frage nach dem Verhältnis zwischen Partikularität und Universalität bedeutet dies, dass das Partikuläre nicht Bruchstück oder Fragment des Ganzen ist, sondern im Partikulären das Universale ganz präsent ist und umgekehrt sich das Universale nur im Partikulären zeigt - und dies eben je verschieden. ${ }^{55}$ Das Universale ist nicht die Summe von Partikularismen, sondern wird via Kommunikation mit und zwischen den Teilen gesichert. Es beschreibt so den relationalen Zusammenhang und Zusammenhalt sowie die gemeinsame Bezogenheit auf eine alle verbindende, gemeinsame Wirklichkeit.

So wie der Begriff der Katholizität seit jeher speziell in Zeiten des Ringens der Kirche um ihr Selbstverständnis reinterpretiert wurde, steht freilich in einer globalen Welt rasanter Diversifizierung eine dogmatische Weiterentwicklung dieser theologischen Idee an: Was bedeutet Katholizität in der Beziehung der Kirche zur religiösen Diversität der Welt?

Für die hier leitende praktische Frage birgt das Modell der Katholizität Potential, die für einen zeit-gerechten Umgang mit Diversität in und außerhalb der Kirche theoretische Grundlagen bieten.

1) Pluralität und Diversität werden als „Normalität“ und Ausgangspunkt des Denkens und Handelns anerkannt. Diversität wird als Partikularität gedacht: d.h. die Verschiedenen sind Teil der einen Menschheit, haben an dieser Teil und brauchen einander. Das Partikuläre ist jedoch weder „Fall“" noch Variante des Ganzen. Das Verhältnis zwischen dem Partikulären und dem Universalen, den Teilen und dem Ganzen ist wechselseitig und komplex. Im Partikulären ist das Universale „ganz" präsent: in der Ortskirche ist die Kirche „ganz“ da, in einem einzelnen Menschen ist die ganze Menschheit $\mathrm{da}^{56}$, in einer gesellschaftlichen Gruppe ist die ganze Gesellschaft präsent. Eine solche Sicht gewährleistet die Würde und Wertschätzung sowie Anerkennung des einzelnen und konkreten Phänomens in seiner Eigenheit.

2) Zugleich bilden das Streben nach Einheit und die Suche nach dem je tieferen Verständnis von Wahrheit den universalen Orientierungshorizont. Einheit und Wahrheit werden als Beziehungswirklichkeiten interpretiert. Sie verwirklichen sich als und in Beziehung auf allen Ebenen: zwischen Individuen, in und zwischen Gruppen, als und in Institutionen. Sie „existieren“ durch

55 Das hat inkarnationstheologische Gründe: Indem Jesus von Nazareth von ChristInnen als der fleischgewordene Logos interpretiert wird, kann damit zugleich alles Konkrete, alles Materielle, Irdische in seiner Partikularität als Ausdruck des Universalen wahrgenommen und wertgeschätzt werden.

56 Dies ist übrigens auch eine Erkenntnis des Talmud und des Koran: Wer einen Menschen rettet, rette die ganze Welt. 
Kommunikation und Teilhabe in der gemeinsamen Suche und Frage nach Einheit und Wahrheit. Auf symbolischer Ebene und in Strukturen werden sie „verdichtet" ausgedrückt. Einheit wird als vorgegebene Wirklichkeit zwischen Menschen verstanden, die zu bewahren und zu gestalten ist: Die Menschen sind konstitutiv untereinander als Menschheit und mit Gott verbunden. Daher sind sie verantwortlich, diese Verbindung zu gestalten; herstellen können sie sie nicht. Wahrheit wird als Beziehung zu einer transzendenten Alterität interpretiert, die als Geheimnis von Menschen nie zur Gänze erkannt und nicht fixiert werden kann. Wahrheit verwirklicht und bewährt sich als Treue zwischen Gott und den Menschen. Einheit „ereignet sich“ als Praxis der Liebe und der Gerechtigkeit.

Eine so verstandene Katholizität könnte dem Kairos einer religiös diversen Welt entsprechen.

\section{Kirchliche Lernorte (Perspektive d)}

Ins Praktische gewendet, bedeutet Katholizität, Vielfalt und Verschiedenheit anzuerkennen. Konkret heißt das: Die Andersheit des/der Anderen als Andersheit und darin auch seine Würde anerkennen. Katholizität praktisch meint, Unterschiede wahrzunehmen, zu beschützen und zu fördern, Partizipation zu eröffnen, sowie für Strukturen der Gerechtigkeit zu sorgen, die die Macht-Ungleichheit zwischen Verschiedenen ausgleichen. Diese praktische Form der Anerkennung von Diversität ist die Basis, auf der Liebe, Gerechtigkeit und die gemeinsame je tiefere Suche nach dem Verständnis von Wahrheit erst möglich werden. Indem dies geschieht, kann die Einheit der Menschen mit Gott und untereinander ein Stückweit mehr wahrnehmbar und wirksam werden. Eine solche Art von Praxis ist aus theologischer Perspektive die via regia zur „katholischen Einheit". Katholizität ist freilich immer schon verletzt und zerstört. Sie ist aus der Sicht des christlichen Glaubens unter endlichen Bedingungen nur im Fragment zu erkennen und zu leben, sie steht als Verheißung in der Zukunft aus. Sie wird anwesend, indem sie als Möglichkeit geglaubt, als Wirklichkeit erhofft und in der eben beschrieben Praxis schrittweise realisiert wird.

Ein solches Verständnis sowie eine so gelebte Praxis von Katholizität ist nach Jahrhunderten einer feudal-hierarchisch, uniform und homogenisierend interpretierten Praxis von Katholizität erst wieder zu gewinnen. Die Aufgabe der Kirche besteht daher darin, in ihren Diözesen und Gemeinden, im Verhältnis zu den anderen christlichen Kirchen, zu anderen Religionen und zur Welt sowie in der Theologie Katholizität zu üben. Für eine Kirche, die sich selbst ungebrochen zum einzigen, zentralen und normativen Bezugspunkt religiöser Wahrheit macht, ist das eine immense Herausforderung. Wohl sind die theologischen Reformen nach dem Zweiten Vatikanum ein riesiger Schritt in Richtung der Wiederge- 
winnung von Katholizität. Aber der Unwille der Katholischen Kirche, z.B. evangelische Kirchen als Kirchen und nicht nur kirchliche Gemeinschaften anzuerkennen oder die Schwierigkeiten, mit der inneren kulturellen, sozialen oder theologischen Vielfalt ${ }^{57}$ umzugehen, machen deutlich, wie schwierig das ist.

Aus praktisch-theologischer Sicht entsteht Veränderung zuerst in der Praxis. Sie ist es, die das Neue zur Welt bringt und immer erst im Nachhinein theologisch ausgelotet werden kann..$^{58}$

Daher stelle ich zum Abschluss einige Beispiele aus der Praxis vor, in denen sich zeigt, dass die Katholische Kirche bereits auf dem Weg ist, zu einem veränderten Umgang mit Diversität zu finden.

1) Im Umgang mit der Diversität der anderen Religionen praktiziert die Katholische Kirche seit dem Zweiten Vatikanischen Konzil einen beeindruckenden interreligiösen Dialog mit den anderen Religionen, der sich in einer Fülle an lehramtlichen Dokumenten niedergeschlagen hat. ${ }^{59}$ Vor allem im Gespräch mit dem Judentum ${ }^{60}$, dessen konstitutive und einzigartige geistliche Bedeutung für das Christentum die Katholische Kirche anerkennt ${ }^{61}$, ist das kirchliche Lehramt der pastoralen Praxis und mitunter auch der katholischen Theologie vielfach weit voraus. Das Wirken von Papst Johannes Paul II. kann in diesem Zusammenhang gar nicht hoch genug veranschlagt werden. Der Reichtum an Handlungsperspektiven ist für das kirchliche Handeln auf lokaler Ebene noch viel zu wenig erschlossen. Auch der globale Einsatz für Gerechtigkeit, Friede und die Bewahrung der Schöpfung, den die Katholische Kirche praktisch und in zahlreichen Dokumenten zur Katholischen Soziallehre bezeugt, kann als Ausdruck der Wertschätzung von Diversität wahrgenommen werden. Die theologische Pionierarbeit und das praktische Engagement der Katholischen Kirche im Kontext von Migration, Flucht und Vertreibung sei in diesem Kontext in besonderer Weise hervorgehoben. Die Anerkennung von innerkirchlicher Diversität zeigt sich z.B. in der Verpflichtung der Ortskirchen, ihre MigrantInnen aufzunehmen und „unbegründeten Verdächtigungen und beleidigenden Vorurteilen gegen die Frem-

57 Man denke in diesem Zusammenhang z. B. an die Konflikte um wiederverheiratete Geschiedene oder das Frauenpriestertum, um Art und Ausmaß der Inkulturation des christlichen Glaubens in afrikanische oder indische Kontexte, an die Konflikte zwischen kirchlichem Lehramt und Befreiungstheologie usw. Alle diese Auseinandersetzungen haben zum Ausschluss von Gläubigen, Theologen, Gruppen aus der Kirche geführt und zeugen von Schwierigkeiten, mit Diversität „katholisch“ umzugehen.

58 Vgl. de Certeau 2009, S. 181.

59 Vgl. Fürlinger 2009.

60 Vgl. Rendtorff / Henrix 1988 und 2001.

61 Dies schlägt sich strukturell darin nieder, dass die Kommission für die religiöse Beziehung mit den Juden seit 1974 mit dem Päpstlichen Rat zur Förderung der Einheit der Christen verbunden ist und nicht beim Päpstlichen Rat für den interreligiösen Dialog. 
den entgegenzutreten “62, in der besonderen Verantwortung der Ortskirche für die Pflege der Sprache und Volksfrömmigkeit ${ }^{63}$ der anderssprachigen Gemeinden, sowie im Anprangern jener Ursachen der globalen Migrationen einer ungerechten, neoliberalen Wirtschaft - die die Vielfalt der Sprachen und Kulturen zerstören.

2) Papst Franziskus ist ein Wegbereiter für eine neue Kultur des Umgangs mit Diversität in Kirche und Welt. Beispielgebend und in der jüngeren Geschichte der Kirche radikal neu ist z. B. sein Führungsverhalten, das Partizipation fördert und an der Vielfalt der Erfahrungen und Meinungen innerhalb der Kirche offensiv interessiert ist. Die Art und Weise, wie er die Familiensynode der Katholischen Kirche (4. bis 25. Oktober 2015) vorbereitete, ist neu für diese Institution. In mehrschleifigen Feedback-Verfahren wurden seit 2013 weltweit mittels eines Fragebogens in jeder Diözese der Kirche die Vielfalt der Erfahrungen und Positionen zu Fragen der (nicht nur pastoralen) Situation von Ehe und Familie vor Ort erhoben. Die Bischöfe waren aufgefordert, ihre Gläubigen vor Ort aktiv in diesen Prozess der Meinungsfindung einzubeziehen. Sie waren ihrerseits bei der Vorbereitungsversammlung - der Außerordentlichen Generalversammlung der Bischofssynode vom 5. bis 19. Oktober 2014 in Rom - angehalten, erstmals frei ihre Meinung kundzutun. Ebenso neuartig für die Kirche war, dass die Diversität der Meinungen der Bischöfe öffentlich kommuniziert wurde, ohne dass der Papst das abschließende „Machtwort“ sprach. Hier kündigt sich nicht nur ein neuer Stil, sondern ein veränderter Umgang mit interner Diversität an. Er könnte es möglich machen, dass die kulturelle Vielfalt der Weltkirche nicht nur sicht- und hörbar werden darf, sondern in einem lehramtlichen Erkenntnis- und Entscheidungsprozess eine konstitutive Rolle bekommt.

Bei der Rede des Papstes vor dem Europarat der Europäischen Union am 25 . November $2015^{64}$ betonte dieser einen globalen, europäischen friedlichen Umgang mit Diversität. So bezeichnet er „Multipolarität“ und „Transversalität" als die beiden wesentlichen Herausforderungen, vor denen Europa steht. „Multipolarität“ bezeichnet dabei seine Vision, ein Europa der pluralen kulturellen, religiösen und politischen Pole zu globalisieren, ohne dabei auf hegemonische Reduktionismen - wie einst „Osten und Westen“ oder „Rom Byzanz - Moskau“ - zurückzugreifen. Die Besonderheit jedes Teils ist dabei zu bewahren. Für die harmonische Einheit des Ganzen ist dabei ebenso zu sorgen wie die konstruktiven Spannungen zwischen diesen Polen zu fördern, die zersetzenden zu beenden. „Transversalität"65 beschreibt die nicht-hintergehbare Pluralität der globalisierten Welt, in der zwischen den verschiedenen

62 Erga Migrantes 41.

63 Erga Migrantes 38; 46.

64 Anspache von Papst Franziskus an den Europarat 2014.

65 Die Erläuterung dieses Begriffes vgl. Kasper 2015. 
ethnischen, kulturellen, religiösen und anderen Gruppen vernünftig kommuniziert und kreativ interagiert wird. Identitäten werden dabei wertgeschätzt und als wechselseitige Bereicherungen verstanden. Der Papst träumt von einer transversalen globalen Identität in einer neuen multipolaren globalen Perspektive. Eine solche Transversalität verlangt „Einfühlungsvermögen“ und „geschichtsbewusste Wachstumsmethodologie" sowie die Bereitschaft, sich nicht in die eigenen Identitäten einzuschließen und ausschließlich innerhalb der eigenen Zugehörigkeitsgruppen zu leben, sondern in ,jugendlichem Geist" zwischen allen Kulturen, Gruppen und Generationen mehrperspektivisch zu dialogisieren.

3) Auch in der Erzdiözese Wien lassen sich Zeichen erkennen, dass die Kirche lernt, mit Diversität umzugehen. Drei Beispiele:

Ein Lernort, der von der Kirche viel zu oft übersehen wird, sind die Entwicklungen im Bereich der Religionspädagogik und des Religionsunterrichts. In der Schule ist Diversität Normalität. Daher liegen in der religionspädagogischen Forschung wie auch in den Erfahrungen des Religionsunterrichtes reiche Schätze vor, wie die Kommunikation des christlichen Glaubens im Kontext von Diversität verwirklicht werden kann. In diesem Zusammenhang sei beispielhaft das Projekt „lebens.werte.schule“66 erwähnt. Dieses entwickelt seit Jahren ein Modell der „Kultur der Anerkennung ${ }^{667}$ in Praxis und Theorie, das sich an den Prinzipien Würde, Gerechtigkeit und Anerkennung orientiert. Die Leitfrage lautet: Wie wird religiöse Diversität wahrgenommen?

Ganz in diesem Sinn versteht sich auch das Schulzentrum Friesgasse ${ }^{68}$ in Wien, eine katholische Privatschule der Schulschwestern. Die Statistik dieses Schulzentrums - 1500 Kinder und Jugendliche leben und lernen in Kindergarten und Hort, Volksschule, Neuer Mittelschule, Gymnasium und Realgymnasium, in einer Handelsschule und einem HAK-Lehrgang - weist pro Jahr ca. 40 verschiedene Muttersprachen und 20 verschiedene Religionsbekenntnisse auf. Von daher hat sich diese Schule aus pädagogischen und weltanschaulichen Gründen in den vergangenen Jahren bewusst multireligiös und multikulturell ausgerichtet und pflegt den interreligiösen Dialog sowie die Ökumene. Konkret wird das Leitbild z. B. in einem regelmäßig kooperierendem Team der Religionslehrerin-

66 Aus dem Leitbild: „Die Initiative lebens.werte.schule sensibilisiert für das Wahrnehmen religiöser Dimensionen in Schulkultur und Schulentwicklung. Eine gute Schule erkennen wir am Umgang mit kultureller und religiöser Pluralität. Unser Ziel ist eine demokratiefähige Schule und ein Beitrag zur menschengerechten Bildung." Die geistigen „Väter“ dieser Initiative sind der katholische Religionspädagoge Martin Jäggle und der evangelische Vizerektor der Kirchlich-Pädagogischen Hochschule Wien - Krems, Thomas Krobath. Vgl. Lebens.werte.schule 2015.

67 Jäggle / Krobath /Stockinger / Schelander 2013.

68 Schulzentrum Friesgasse 2015. 
nen. Die Diversität der Schülerinnen und Schüler wird öffentlich sichtbar, wenn man die Schule betritt: Transparente begrüßen den Gast mehrsprachig, kulturelle und religiöse Symbole verschiedenster Traditionen sind sichtbar. Dass die Betriebsküche auf unterschiedliche religiöse Ernährungstraditionen Rücksicht nimmt, ist selbstverständlich. In diese Schule schicken auch muslimische Eltern ihre Kinder gerne, weil ihre Religion dort anerkannt und unterrichtet wird.

Auch die Pfarren des zweiten Wiener Gemeindebezirkes ${ }^{69}$ - ein Bezirk mit hohem MigrantInnenanteil, zahlreichen orthodoxen Kirchen, Moscheen und Synagogen - haben begonnen, ihr „Programm“ im Umgang mit der Diversität ihres Bezirkes entsprechend neu auszurichten. Vom Herbst 2014 bis zum Frühjahr 2015 fand eine Umfrage statt, die eruieren sollte, was sich die Bezirksbevölkerung von der Kirche in diesem Bezirk erwartet. Eine erste Auswertung der Ergebnisse zeigt ähnlich wie die Umfrage des Zukunftsforums3000, dass die Befragten die wachsende Diversität des Bezirkes deutlich wahrnehmen. Die Erwartungen an die Kirche sind allerdings widersprüchlich. Ängste werden geäußert, dass der zweite Bezirk „seinen Charakter durch Zuwanderung verliert ${ }^{\text {“70 }}$ und die Kirchen wieder „mehr auf Mission setzen“ sollen, damit „das Katholische nicht verloren geht“. „Leider sind wir kein deutscher Bezirk mehr, sondern ein Ort mitteleuropäischer, vorderorientalischer Prägung mit entsprechender Bevölkerung [...]“ klagt ein Teilnehmer der Befragung. „Christliche Anstandsregeln“ sollten daher wieder gefördert werden. Andere Befragte wiederum wünschen sich, dass sich die Pfarren stärker mit den anderen Religionsgemeinschaften vernetzen und Inklusion fördern. Mancher erkennt den Zuzug als „,soziale und kulturelle Bereicherung“ und „Kultimulti“ ( sic!) könne zur „Normalität“ werden. Der zweite Bezirk sei „ein Schmelztiegel für die Zukunft" und die Kirche solle sich daher für interreligiöse/interkulturelle Treffs und Dialog stark machen. Interkulturelle Kompetenz solle gefördert werden, die Kirche „kann die Chance nutzen, sich mit der Bevölkerung mitzuentwickeln, Anlaufstelle für alle Generationen sein, Trends wie Naturverbundenheit, Nachhaltigkeit, Tauschen statt Neues kaufen, anderen Zeit schenken, Themen wie Erziehung, Familie, Umgang mit älteren Menschen“ aufgreifen. Ein Teilnehmer schreibt: „Pluralismus bei den Religionen bringt immer auch Ängste mit sich - das Fremde bleibt bedrohlich, bis man es kennen lernt - darin liegen die Chancen: Aktiv und mutig aufeinander zugehen und über die Kulturen ins Gespräch kommen und ggf. Anteil nehmen. [...] Kirche ist dann positiv erlebbar, wenn sie offen ist für Menschen aller Religionen und Kulturen und die Botschaft, die sie zu verkünden hat als Angebot bereit hält und die Entscheidung dafür, mitzumachen, mitzugehen, mitzudenken, mitzuleben,

69 Pfarre Machstrasse 2014.

70 Die zitierten Aussagen entstammen den Fragebögen, die mir von einem Pfarrgemeinderat dankenswerter Weise zur Einsicht gegeben wurden. Sprach-, Schreib- und Interpunktionsfehler wurden nicht korrigiert. 
den einzelnen Menschen überlässt, weil sie darauf vertraut, dass Gott wirkt, wenn wir unsere Begeisterung zeigen!“

Hier wartet eine Menge Arbeit auf die Kirche des zweiten Wiener Gemeindebezirkes: der Diversität der Wahrnehmungen und Erwartungen gerecht zu werden und diese aus der Sicht des christlichen Glaubens so zu deuten, dass sie in Entschiedenheit eine Praxis finden kann, die den Menschen vor Ort beim Zusammenleben in Diversität hilft.

\section{Schluss}

In vier Schritten habe ich gezeigt, dass das Thema religiöse Diversität für die Katholische Kirche zuallererst ein Thema ist, mit dem sie sich praktisch, politisch, theologisch und vor allem innerkirchlich auseinandersetzen muss, wenn sie ihrem Wesen und Auftrag gerecht werden möchte. Dazu gehört 1) das Wahrnehmen des globalen wie sozioreligiösen Kontextes, in dem sich religiöse Diversifizierungsprozesse dynamisieren sowie die selbstkritische Auseinandersetzung mit der binnenkirchlichen Aggression gegen „Andere“ und Fremdheitserfahrungen auBerhalb und innerhalb der Kirche. 2) Die aktuelle weltpolitische Lage sowie das historische Erbe verpflichten die Kirche ebenso dazu, sich dieser Thematik offensiv zuzuwenden wie das kirchliche Selbstverständnis als „Zeichen und Werkzeug“ für „Einheit in Vielfalt“. 3) Theologisch stehen zur Auseinandersetzung religiöser Diversität neu zu erschließende bibeltheologische Traditionen zur Deutung von Diversität, das systematisch-theologische Modell der „Katholizität“ sowie lehramtliche Schreiben im Bereich der Migrationstheologie sowie der Katholischen Soziallehre zur Verfügung. Schließlich zeigt 4) ein exemplarischer Blick auf Entwicklungen in der Praxis (hier am Beispiel der Dogmatik des Zweiten Vatikanums, der Praxis von Papst Franziskus und der Erzdiözese Wien), dass die Kirche bereits dabei ist, den Umgang mit Diversität neu zu lernen. Die Kirche verfügt also durchaus über theologische Traditionen sowie - freilich theologisch noch fruchtbar zu machende - praktische Erfahrungen für einen anerkennenden, gerechten und friedlichen Umgang mit Diversität.

\section{Bibliographie}

Agentur der Europäischen Union für Grundrechte: Diskriminierung und Hasskriminalität gegenüber Juden in den EU-Mitgliedstaaten, 2014, abrufbar unter: http://fra. europa.eu/de/publication/2014/diskriminierung-und-hasskriminalitat-gegenuber-ju den-den-eu-mitgliedstaaten [07.06.2015].

Agentur der Europäischen Union für Grundrechte: Muslime in der Europäischen Union, Diskriminierung und Islamophobie, 2006, abrufbar unter: http://fra.europa.eu/sites/ default/files/fra_uploads/156-Manifestations_DE.pdf [07.06.2015]. 
Anderssprachige Gemeinden in Wien, 2015, abrufbar unter: http://www.erzdioezesewien.at/site/menschenorganisation/pfarrenordengemeinschafte/anderssprachigege meinden [07.06.2015].

Arts, Will / Halman, Loek: ,Value Research and Transformation in Europe', in: Polak, Regina (Hg.): Zukunft. Werte. Religion. Die europäische Wertestudie 1990-2010: Österreich im Vergleich. Wien 2011, S. 79-99, S. 99, S. 86.

Baier, Karl: ,Spiritualitätsforschung heute', in: Baier, Karl (Hg.): Handbuch Spiritualität. Zugänge, Traditionen, Interreligiöse Prozesse. Darmstadt 2006, S. 11-45, S. 13.

Bodenheimer, Alfred: Jüdische Migration in, nach und aus Europa', in: Polak, Regina / Reiss, Wolfram (Hg.): Religion im Wandel. Transformation religiöser Gemeinschaften in Europa durch Migration - Interdisziplinäre Perspektiven. Wien 2014, S. 389 396.

Burchardt, Marian / Wohlrab-Sahr, Monika: ,Über die kulturellen Bedeutungen religiössäkularer Kontroversen in der Gegenwart‘, in: Buß, Gregor / Luber, Markus (Hg.): Neue Räume öffnen. Mission und Säkularisierung weltweit. Regensburg 2013, S. 33 55.

Buß, Gregor / Luber, Markus (Hg.): Neue Räume öffnen. Mission und Säkularisierung weltweit. Regensburg 2013.

Bünker, Arnd: ,Migrationsgemeinden als Sehhilfe. Überlegungen zur veränderten Realität des Christlichen in Mitteleuropa', in: Bitter, Gottfried / Blasberg-Kuhnke, Martina (Hg.): Religion und Bildung in Kirche und Gesellschaft. Festschrift für Norbert Mette. Würzburg 2011, S. 85-92.

de Certeau, Michael: ,Der gründende Bruch‘, in: ders.: GlaubensSchwachheit. Stuttgart 2009, S. 155-187, S. $181 \mathrm{f}$.

Collet, Giancarlo: ,Gemeinsam das Evangelium verkünden', in: Bünker, Arnd / Mundanjohl, Eva / Weckel, Ludger / Suermann, Thomas (Hg.): Gerechtigkeit und Pfingsten. Viele Christentümer und die Aufgabe einer Missionswissenschaft. Ostfildern 2010, S. 242-266, S. 242-266.

Ebach, Jürgen: ,Globalisierung - Rettung der Vielfalt. Die Erzählung vom „Turmbau zu Babel" im aktuellen Kontext', in: Schröter, Hartmut (Hg.): Weltentfremdung, Weltoffenheit, Alternativen der Moderne: Perspektiven aus Wissenschaft - Religion Kunst. Münster 2008, S. 39-58.

Evangelische Kirche A.B. und H.B. in Österreich: Internationale Gemeinden der Evangelischen Kirche in Wien, 2015, abrufbar unter: http://www.evang-wien.at/index. php id=33 [07.06.2015].

Freikirchen in Österreich, 2015, abrufbar unter: http://www.freikirchen.at/ [07.06.2015].

Gauthier, François / Martikainen, Tuomas (eds.): Religion in Consumer Society. Farnham 2013.

Fürlinger, Ernst (Hg.): Der Dialog muss weitergehen. Ausgewählte vatikanische Dokumente zum interreligiösen Dialog (1964-2008). Freiburg i. B. 2009.

Goujon, Anne u.a.: Past present and future prospects in Vienna 1950-2050, 2015, abrufbar unter: http://vidwirel.oeaw.ac.at/; http://witt.null2.net/wireldataviz/ [07.06.2015].

Hafez, Kai / Schmidt, Sabrina: Die Wahrnehmung des Islams in Deutschland. (Religionsmonitor Sonderauswertung der Bertelsmann-Studie). Gütersloh 2015. 
Höllinger, Franz / Tripold, Thomas: Ganzheitliches Leben. Das holistische Milieu zwischen neuer Spiritualität und postmoderner Wellness-Kultur. Bielefeld 2012.

Hrvatska Katholička Misija Beč (Kroatisch-Katholische Mission in Wien), 2015, abrufbar unter: http://www.hkm-wien.at/joomla/index.php [07.06.2015].

Identity Foundation: Spiritualität in Deutschland, 2006, abrufbar unter: http://www. kleine-spirituelle-seite.de/tl_files/template/pdf/studie_spiritualitaet_in_deutschland. pdf; http://zelos.zeit.de/bilder/2006/15/aktuell/Studie_Spiritualitaet.pdf [07.06.2015].

Jäggle, Martin / Krobath, Thomas / Stockinger, Helena / Schelander, Robert (Hg.): Kultur der Anerkennung. Würde - Gerechtigkeit - Partizipation für Schulkultur, Schulentwicklung und Religion. Hohengehren 2013.

Kasper, Walter: Papst Franziskus - Revolution der Zärtlichkeit und der Liebe. Stuttgart 2015.

Katholische Aktion Österreich 2015a: Zukunftsforum3000, 2013, abrufbar unter: http:// www.zukunftsforum3000.at/site/home [07.06.2015].

Katholische Aktion 2015b: Über das Projekt, 2013, abrufbar unter: http://www.zu kunftsforum3000.at/site/ueberdasprojekt [07.06.2015].

Kuschel, Karl-Josef: Juden, Christen, Muslime. Herkunft und Zukunft. Ostfildern, 3. Auflage, 2007, 234-249. 303-305.

Lebens.werte.schule, 2015, abrufbar unter: http://lebenswerteschule.univie.ac.at/.

Maalouf, Amin: Die Auflösung der Weltordnungen, Berlin 2010, S. 27.

Martikainen, Tuomas: Migration and Contemporary Religious Change, 2015, abrufbar unter: http://vidwirel.oeaw.ac.at/conference/ [07.06.2015].

Orthodoxe Kirche in Österreich, 2015, abrufbar unter: http://www.orthodoxe-kirche.at/ [07.06.2015].

PEW-Research-Center's Forum on Religion and Public Life: Faith on the Move: The Religious Affiliation of International Migrants, 2012, abrufbar unter: http://www. pewforum.org/2012/03/08/religious-migration-exec/ [07.06.2015].

PEW-Research-Center's Forum on Religion and Public Life: Global Religious Diversity, 2014, abrufbar unter: http://www.pewforum.org/2014/04/04/global-religious-diversity [07.06.2015].

PEW-Research-Center's Forum on Religion and Public Life: The Future of World Religions: Population Growth Projections 2010-2050, 2015, abrufbar unter: http://www. pewforum.org/2015/04/02/religious-projections-2010-2050/ [07.06.2015].

PEW-Research-Center's Forum on Religion and Public Life: The global religious landscape, 2012a, abrufbar unter: http://www.pewforum.org/2012/12/18/global-reli gious-landscape-exec/ [07.06.2015].

Pfarre Machstrasse: Online-Umfrage, 2014, abrufbar unter: http://www.pfarre-mach strasse.at/Aktuell/Befragung.htm [24.11.2014].

Polak, Regina: ,Migration und Katholizität‘, in: Polak, Regina / Reiss, Wolfram (Hg.): Religion im Wandel. Transformationsprozesse religiöser Gemeinschaften in Europa durch Migration - Interdisziplinäre Perspektiven. Reihe: Religion and Transformation in Contemporary European Society 9. Wien 2014, S. 233-296.

Polak, Regina: „,Wir sind keine Indianer!‘ - Erfahrungen der kroatisch-katholischen Gemeinde in Wien“, in: International Journal for Practical Theology 2015/2, in Erscheinung. 
Polak, Regina: Religiosität und Migration. Eine qualitativ-empirische Studie. Erste Ergebnisse. Unveröff. Bericht. Wien 2014.

Rendtorff, Rolf / Henrix, Hans-Hermann (Hg.): Die Kirchen und das Judentum. Dokumente von 1945 bis 1985, Paderborn und München 1988.

Rendtorff, Rolf / Henrix, Hans-Hermann (Hg.); Die Kirchen und das Judentum. Dokumente von 1986-2000. Paderborn und Gütersloh 2001.

Rosenberger, Sieglinde / Seeber, Gilg: ,Kritische Einstellungen: BürgerInnen zu Demokratie, Politik, Migration', in: Polak, Regina (Hg.): Zukunft. Werte. Religion. Die europäische Wertestudie 1990-2010: Österreich im Vergleich. Wien 2011, S. 165-189.

Schulzentrum Friesgasse, 2015, abrufbar unter: http://www.schulefriesgasse.ac.at/ [07.06.2015].

Stadt Wien - Magistratsabteilung 17 - Integration und Diversität (Hg.): Integrations- und Diversitätsmonitor der Stadt Wien 2009-2011, 2011, abrufbar unter: http://www.wien. gv.at/menschen/integration/pdf/monitor-2012.pdf [07.06.2015].

Stolz, Jörg / Könemann, Judith / Schneuwly Purdie, Mallory / Englberger, Thomas / Krüggeler, Michael: Religion und Spiritualität in der Ich-Gesellschaft. Zürich 2014.

Zukunftsforum der Katholischen Kirche in Österreich: Online-Umfrage 2013. Bericht, 2015, abrufbar unter: http://www.wodruecktderschuh.at/site/umfrage/article/21.html [07.06.2015].

Vencser, Laszlo / Scharl, Franz (Hg.): Einheit in Vielfalt. 40 Jahre Nationaldirektion der katholischen fremdsprachigen Seelsorge in Österreich 2015. Linz 2015.

\section{Kirchliche Dokumente}

Ansprache von Papst Franziskus an den Europarat, 25. November 2014, abrufbar unter: https://w2.vatican.va/content/francesco/de/speeches/2014/november/documents/papafrancesco_20141125_strasburgo-consiglio-europa.html [07.06.2015].

Dignitatis Humanae. Erklärung zur Religionsfreiheit, 1965, abrufbar unter: http://www. vatican.va/archive/hist_councils/ii_vatican_council/documents/vat-ii_decl_19651207_di gnitatis-humanae_ge.html [07.06.2015].

Erga migrantes caritas Christi. Instruktion des Päpstlichen Rates der Seelsorge für die Migranten und Menschen unterwegs, 2004, abrufbar unter: http://www.vatican.va/ roman_curia/pontifical_councils/migrants/documents/rc_pc_migrants_doc_20040514_ erga-migrantes-caritas-christi_ge.html [07.06.2015].

Lumen Gentium. Dogmatische Konstitution Lumen Gentium über die Kirche, 1962, abrufbar unter: http://www.vatican.va/archive/hist_councils/ii_vatican_council/docu ments/vat-ii_const_19641121_lumen-gentium_ge.html [07.06.2015].

Nostra Aetate. Erklärung über das Verhältnis der Kirche zu den nichtchristlichen Religionen, 1965, abrufbar unter: http://www.vatican.va/archive/hist_councils/ii_vatican_ council/documents/vat-ii_decl_19651028_nostra-aetate_ge.html [07.06.2015].

Unitatis Redintegratio. Dekret über den Ökumenismus, 1964, abrufbar unter: http:// www.vatican.va/archive/hist_councils/ii_vatican_council/documents/vat-ii_decree_ 19641121_unitatis-redintegratio_ge.html [07.06.2015]. 